\title{
AVALIAÇÃO DO ESTADO NUTRICIONAL DOS BENEFICIÁRIOS ADULTOS DO PROGRAMA BOLSA FAMÍLIA DA CIDADE DE DOURADOS-MS
}

\section{EVALUATION OF THE NUTRITIONAL STATUS OF THE ADULT BENEFICIARIES OF THE FAMILY BAG PROGRAM IN THE CITY OF DOURADOS-MS}

\author{
Dayane Ribeiro Dos Santos ${ }^{1}$; Patricia Cintra².
}

${ }^{1}$ Nutricionista formada pelo Centro Universitário da Grande Dourados - UNIGRAN, Dourados - MS, Brasil.

${ }^{2}$ Docente do curso de Nutrição, Centro Universitário UNIGRAN CAPITAL, Campo Grande - MS, Brasil. 


\section{RESUMO}

Objetivo: Esta pesquisa teve como objetivo avaliar o estado nutricional em adultos usuários do Programa Bolsa Família. Métodos: A pesquisa foi de caráter quantitativo, descritivo com delineamento retrospectivo. Foi avaliado o estado nutricional dos usuários do Programa Bolsa Família utilizando os marcadores: índice de massa corporal (IMC) e avaliação do consumo alimentar com o auxílio do questionário de consumo do SISVAN. Os dados da pesquisa foram coletados dos relatórios do SISVAN de uso privado e a população avaliada envolveu 380 indivíduos adultos. Foram avaliados a prevalência de sobrepeso e obesidade nessa população entre os anos de 2016 e 2017, entre os marcadores de consumo alimentar saudáveis foram avaliados: o consumo de feijão, frutas, verduras e legumes e dos marcadores não recomendáveis foram avaliados os alimentos ultraprocessados. Resultados: Foram encontradas altas prevalências de sobrepeso e obesidade no presente estudo, destacando 0 alto consumo de alimentos ultraprocessados e a diminuição do consumo de feijão, frutas, legumes e verduras.

Palavras-chave: programa bolsa família, avaliação nutricional, consumo alimentar, sobrepeso, obesidade. 


\section{INTRODUÇÃO}

Toda a população tem direito à alimentação e nutrição adequadas, ou seja, estar livre da fome e da má nutrição seja por seus próprios recursos ou por ajuda do governo. ${ }^{1}$

O Bolsa Família é um programa de transferência direta de renda, criado pela lei № 10.836 , de 9 de janeiro de 2004. O programa é regulamentado pelo Decreto $n^{\circ} 5.209$, de 17 de setembro de 2014, para famílias em situação de extrema pobreza para garantir seu acesso aos direitos à assistência social, à educação e a saúde também, sendo distribuído um benefício financeiro de acordo com cada família. ${ }^{2,3,1}$

Uma das funcionalidades é garantir esse acesso, pois essas famílias em situação de pobreza e extrema pobreza têm uma maior dificuldade de acesso e frequência em postos de saúde, escolas, por isso o objetivo é garantir esse acesso, melhorar a qualidade de vida dessas famílias e contribuir na inclusão social. ${ }^{4}$

A operacionalização deste programa consiste no repasse mensal de benefícios em dinheiro para as famílias cadastradas. São quatros tipos de valores que variam de acordo com a característica de cada família, são eles: o benefício básico consiste no valor repassado de $R \$ 85,00$ e é para famílias com renda mensal de até $R \$ 85,00$ per capita, mesmo que essa família não possua crianças, adolescentes, jovens, gestantes ou nutrizes. No benefício variável é repassado o valor de $R \$ 39,00$ e é concedido para famílias com renda mensal de até $R \$ 170,00$ per capita, porém devem conter crianças, adolescentes de até 15 anos, gestantes e/ou nutrizes. ${ }^{5}$ 
Para as gestantes esse valor repassado ocorre por nove meses, a partir do registro do acompanhamento do pré-natal, e para as nutrizes o valor é repassado durante 6 meses a partir da inclusão do recém-nascido no cadastro único. Portanto, cada família pode receber até cinco benefícios variáveis, no máximo, sendo que essa família pode receber até $R \$ 195,00$ por mês. ${ }^{5}$

O benefício variável vinculado ao adolescente (BVA) é concedido o valor de $R \$ 47,00$ a todas as famílias que contenham adolescentes de 16 e 17 anos e que frequentem a escola, no entanto, a família pode receber até dois benefícios variáveis ao adolescente, totalizando até $R \$ 94,00$ até o jovem completar 18 anos. ${ }^{5}$

Finalizando o benefício para superar a extrema pobreza na primeira infância não é fixo, ele é calculado de acordo com a necessidade cada família, para que as mesmas consigam superar a situação de extrema pobreza e ultrapasse a renda do benefício básico por pessoa. ${ }^{5}$

A avaliação nutricional é o primeiro passo no processo do cuidado nutricional. Para desenvolver um bom plano nutricional, a avaliação deve incluir os principais elementos como: histórico médico do indivíduo, medidas antropométricas, o problema atual, valores laboratoriais e bioquímicos, uso de medicamentos e suplementos, além do histórico da alimentação e a ingestão de nutrientes. ${ }^{6}$

O Sisvan é um sistema que compõe os registros do acompanhamento do estado nutricional e dos marcadores do consumo alimentar das pessoas atendidas no âmbito da atenção básica. Para os beneficiários do Programa Bolsa Família (PBF) é uma condicionalidade que o município oferta para que eles possam ter os mesmos direitos à saúde. Todos os dados do 
acompanhamento nutricional dos beneficiários do PBF são feitos pelo Sisvan e são semestralmente importados para o Sisvan Web no final de cada vigência, sendo a primeira vigência de janeiro a junho, e segunda vigência de julho a dezembro. ${ }^{7,8}$

Diante dessas considerações, este estudo teve como objetivo avaliar o estado nutricional por meio do índice de massa corporal e avaliar o consumo alimentar com auxílio do questionário do Sisvan dos adultos beneficiários do programa Bolsa Família na cidade de Dourados-MS.

\section{METODOLOGIA}

A pesquisa foi de caráter quantitativa, descritiva com delineamento retrospectivo com referenciais teóricos cujo objetivo é obter resposta para 0 problema, no caso em questão identificar a prevalência de sobrepeso e obesidade entre os usuários do bolsa família. Este tipo de pesquisa apresenta métodos para testar as hipóteses numericamente, podendo gerar valores para serem comparados e discutidos com outras pesquisas quantitativas. Já a pesquisa descritiva tem como objetivo descrever as características de uma determinada população com objetivo final de obter e confirmar uma resposta ou não. Para finalizar, o estudo retrospectivo é quando são coletados informações do passado, por meio de exames registrados, prontuários e entrevistas. ${ }^{9}$

A pesquisa procedeu por meio de coleta de dados de relatórios do SISVAN da cidade de Dourados, município brasileiro da região Centro-Oeste, no estado do Mato Grosso do Sul, habitada por aproximadamente 220 mil habitantes. 
Os dados foram coletados do Sistema de Vigilância Alimentar e Nutricionais (SISVAN) (Anexo A), por meio do acesso privado, referentes ao estado nutricional e consumo alimentar dos adultos beneficiários, sendo autorizado o acesso pela Secretaria Municipal de Saúde de Dourados.

A população escolhida para a pesquisa foram beneficiários adultos cadastrados no Bolsa Família e SISVAN web na cidade de Dourados - MS no período de 2016 e 2017, do sexo masculino e feminino, com idade de 20 a 59 anos completos.

Foram excluídos da pesquisa crianças, adolescentes, idosos, gestantes, indígenas e os mesmos que não são beneficiários do Programa Bolsa Família (PBF) e que não possuía cadastro no SISVAN web.

Foi realizado o termo de guarda das informações (Apêndice A), a fim de preservar a privacidade dos pacientes cujos dados antropométricos e de consumo alimentar foram coletados em relatórios no sistema do Sisvan de indivíduos cadastrados na Bolsa Família e no Sisvan web.

A coleta de dados do SISVAN foi autoriza pela secretaria municipal da Saúde de Dourados - MS (Anexo B).

Para classificação do estado nutricional de adultos, o Ministério da Saúde adota os pontos de corte para o índice de massa corporal (IMC) calculado por meio do peso do indivíduo (em $\mathrm{kg}$ ) dividido pela altura (em metro) ao quadrado - recomendado pela Organização Mundial da Saúde (1997), (Tabela 1). 
Tabela 1: Classificação do IMC segundo a OMS.

\begin{tabular}{|c|c|}
\hline$<16$ Magreza grau III & 16 a 16.9 Magreza grau II \\
\hline 17 a 18.4 Magreza grau I & 18.5 a 24.9 Adequado \\
\hline 25 a 29.9 Pré - obeso & 30 a 34.9 Obesidade grau I \\
\hline 35.0 a 39.9 Obesidade grau II & $>=40$ Obesidade grau III \\
\hline
\end{tabular}

Fonte: Organização Mundial da Saúde $(1995,1997) .{ }^{10}$

O relatório gerado pelo SISVAN apresenta o número de indivíduos e a porcentagem correspondente para cada estado nutricional classificado pelo IMC e também pelo sexo, ou seja, masculino e feminino.

O peso e altura registrados no SISVAN web, são exportados do bolsa família no final de cada vigência do programa, onde são enviados os mapas das famílias que devem ser acompanhados para as unidades básicas de saúde e devem ser preenchidos e devolvidos até a data estabelecida pela Secretaria Municipal de Saúde de Dourados, para que possam ser registrados esses dados no site do Programa Bolsa Família.

Posteriormente, são gerados os relatórios de consumo alimentar na página do módulo gerador de relatórios do Sisvan.

Para cada questão, no formulário consta o questionamento do seu consumo no dia anterior, com as opções de resposta: sim, não e não sabe. No relatório de cada questão, gerado pelo SISVAN, é apresentado o número de indivíduos que referiu o consumo e seu valor percentual de acordo com o número total de entrevistados. O questionário é baseado em 9 questões, porém para o estudo realizado foram descartadas duas questões e divididos em Marcadores saudáveis e Marcadores não Saudáveis. 
Todos os relatórios gerados pelo SISVAN foram exportados para a Microsoft Excel e, posteriormente, organizados para a tabulação dos resultados, no qual foram avaliados pelo método de porcentagem, média e desvio padrão, no qual o erro amostral foi utilizado à fórmula de Barbetta (2002). ${ }^{11}$

\section{RESULTADOS E DISCUSSÃO}

Foram avaliados 137 indivíduos adultos do Programa Bolsa Família no ano de2016 e 205 indivíduos em 2017 de ambos os sexos, resultando em 372 indivíduos avaliados. Dentre estes, 69,34\% eram mulheres e 30,65\% homens. A (Tabela 2) descreve a distribuição da renda das famílias beneficiadas.

Tabela 2- Famílias com renda mensal de $R \$ 85,01$ a $R \$ 170,00$ por pessoa.

\begin{tabular}{cccc}
\hline $\begin{array}{c}\text { Número de } \\
\text { gestantes, } \\
\text { nutrizes, crianças } \\
\text { e adolescentes até } \\
\mathbf{1 5} \text { anos. }\end{array}$ & $\begin{array}{c}\text { Número de jovens } \\
\text { de 16 e 17 anos }\end{array}$ & Tipo de benefício & $\begin{array}{c}\text { Valor do } \\
\text { benefício }\end{array}$ \\
\hline $\mathbf{0}$ & 0 & & \\
$\mathbf{1}$ & 0 & Não recebe & \\
$\mathbf{2}$ & 0 & benefício & - \\
$\mathbf{0}$ & 1 & 2 variável & $\mathrm{R} \$ 39,00$ \\
$\mathbf{1}$ & 1 & 1 BVJ & $\mathrm{R} \$ 78,00$ \\
$\mathbf{2}$ & 1 & 1 variável $+1 \mathrm{BVJ}$ & $\mathrm{R} \$ 47,00$ \\
$\mathbf{0}$ & 2 & 2 variáveis $+1 \mathrm{BVJ}$ & $\mathrm{R} \$ 125,00$ \\
$\mathbf{3}$ & 2 & 2 BVJ & $\mathrm{R} \$ 94.00$ \\
\hline
\end{tabular}

Fonte: Secretaria Municipal de Assistência Social, $2016 .{ }^{5}$ 
A (Tabela 3) descreve o estado nutricional da população avaliada pelo Índice de Massa Corporal (IMC), sendo esses valores apresentados à soma do ano de 2016 e 2017, gerando esse valor descrito, 6,64\% baixo peso, $63,02 \%$ eutrófico, $70,86 \%$ sobrepeso e $59,43 \%$ obeso. Podendo ser percebido a prevalência de sobrepeso e obesidade em maior número no estudo.

Tabela 3- Classificação do Estado Nutricional do ano de 2016 e 2017 de ambos os sexos.

\begin{tabular}{llrlr}
\hline $\begin{array}{llll}\text { Classificação } \\
\text { Nutricional }\end{array}$ & $\begin{array}{l}\mathbf{2 0 1 6} \\
(\mathbf{n = 8 4 )}\end{array}$ & & $\begin{array}{l}\mathbf{2 0 1 7} \\
(\mathbf{n = 5 3 )}\end{array}$ & \\
& $\mathbf{N}$ & $\%$ & $\mathbf{N}$ & $\%$ \\
\hline Baixo Peso & 4 & 4,76 & 1 & 1,88 \\
Eutrofia & 26 & 30,95 & 17 & 32,07 \\
Sobrepeso & 31 & 36,90 & 18 & 33,96 \\
Obesidade Grau I & 11 & 13,09 & 8 & 15,09 \\
Obesidade Grau II & 9 & 10,71 & 4 & 7,54 \\
Obesidade Grau III & 3 & 3,57 & 5 & 9,43 \\
\hline
\end{tabular}

Fonte: Autores.

Um estudo no município de Curitiba com o mesmo delineamento experimental verificou em 2011, 747 indivíduos. O objetivo foi verificar o estado nutricional da população adulta beneficiaria do programa bolsa família, relacionando as variáveis socioeconômicas. O estudo demonstrou que $88 \%$ eram mulheres e $12 \%$ homens, desse universo $3 \%$ eram baixo peso e $56 \%$ representaram sobrepeso e obesidade demonstrando a transição nutricional que vem ocorrendo no país. ${ }^{12}$

Em 2013 foram avaliados as variáveis econômicas de 847 indivíduos do PBF, tendo como objetivo avaliar o estado nutricional, o consumo e a segurança alimentar dos beneficiários das favelas de Maceió- AL. Dessa 
população 326 eram adultos, sendo $38,7 \%$ homens e $61,3 \%$ mulheres, dentre esses, $51,2 \%$ apresentaram excesso de peso e $48 \%$ eram mulheres. ${ }^{13}$

Um estudo realizado no município de Montes Claros - MG, com o objetivo de diagnosticar a situação de insegurança alimentar e nutricional entre os beneficiários do PBF, avaliou as variáveis sociodemográficas e foi demonstrado que $59,7 \%$ estavam inadequados, com diagnóstico de sobrepeso ou obesidade. Essa população também não possuia conhecimento sobre alimentação. $^{14}$

Segundo o Ministério da Saúde houve aumento na prevalência de excesso de peso, chegando em $53,8 \%$ dos adultos com excesso de peso e $18,9 \%$ com obesidade, sendo que o número de casos de obesidades é mais elevado nas famílias com baixa escolaridade e o número de casos com excesso de peso é maior conforme a idade avança e também com baixa escolaridade. ${ }^{15}$

Portanto, o presente estudo demonstra informações relevantes, mostrando a alta prevalência de sobrepeso e obesidade na população beneficiária, sendo que essa condição do estado nutricional está associada a um maior risco de doenças crônicas não transmissíveis. Esses dados quando comparados com a população que não recebe o benefício, também demonstram altas prevalências, apontando a transição nutricional que ocorreu e ainda ocorre no país. As alterações que ocorreram e que ainda ocorrem no país foram a respeito da qualidade e quantidade da dieta, mudanças no estilo de vida, mudanças socioeconômicas e demográficas acarretando em altas prevalências de sobrepeso e obesidade e doenças crônicas não transmissíveis independentes de idade, sexo ou classe social. Foi observado que essa 
transição nutricional no país foi devido ao aumento no consumo de gorduras saturadas, açúcares simples, refrigerantes, produtos industrializados, doces em geral e chocolates. Em contra partida houve diminuição no consumo de frutas, verduras e legumes. ${ }^{16}$

O (Gráfico1) demonstra os marcadores alimentares dos 372 adultos beneficiários do PBF avaliados entre os anos de 2016 e 2017 de ambos os sexos. Em relação ao consumo de leguminosa a diminuição do consumo foi de $5,34 \%$, enquanto o grupo de frutas obteve $3,54 \%$. Quanto ao consumo de verduras e Legumes notou-se uma redução de 1,24\%.

Gráfico 1- Distribuição percentual dos indivíduos adultos cadastrados pelo PBF e pelo SISVAN que referiam consumo regular conforme Marcadores Saudáveis Recomendados, no município de Dourados -MS, no ano de 2016 e 2017.

\section{Marcadores de Alimentos Recomendados}

$$
=2016(\%)=2017(\%)
$$
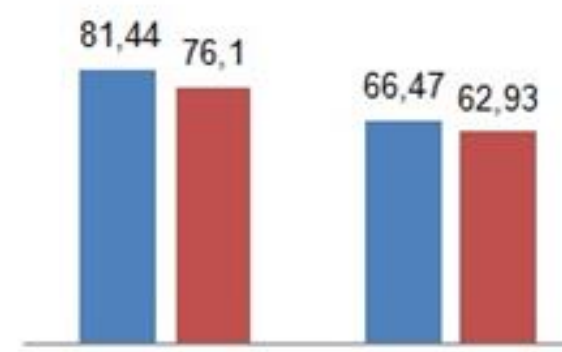

$72,4671,22$

Feijão

Frutas

Verduras e legumes

Fonte: Autores.

De acordo com essa informação, no ano de 2017 houve um aumento na questão da população avaliada. Neste gráfico encontram-se os marcadores de alimentos recomendados: o consumo de feijão, frutas, verduras e legumes e sua porcentagem em relação ao consumo desses pelos usuários adultos do PBF. 
Um diagnóstico de Insegurança Alimentar e Nutricional revelou, a partir de um estudo sócio demográfico que avaliou o município de Montes Claros MG, que 72 indivíduos do PBF apresentaram inadequação no consumo de marcadores saudáveis. Foi constatado o não consumo de verduras e legumes $(52,6 \%)$, leguminosas $\left(53,8 \%\right.$ e cereal $(51,6 \%)$ respectivamente. ${ }^{14}$

Para avaliar o consumo alimentar de usuários do Sistema Único de Saúde, foram avaliados 187 indivíduos, na cidade de Porto Alegre-RS no período de novembro de 2012 a maio de 2013. Foi desenvolvido o questionário com os dados pessoais e a avaliação do consumo alimentar foi feito pelo questionário do SISVAN. Foi identificado que $40(21,4 \%)$ possuíam o benefício do Bolsa Família e 80\% eram mulheres. Levando em consideração todos os participantes, $41 \%$ apresentaram consumo saudável de alimentos e a frequência de consumo alimentar saudável foi: hortaliças (74\%), feijão (72\%) e frutas $(68 \%) .{ }^{17}$

No estudo que avaliou o PBF na região Nordeste e Sudeste, foi detectado que $68 \%$ do total de ingestão de energia dos beneficiários foram na forma de alimentos in natura ou minimamente processados. Para avaliação foi utilizado o Guia Alimentar da população brasileira e foi verificado que os beneficiários do programa estão atendendo as recomendações do Guia. ${ }^{18}$

O IBASE (Instituto Brasileiro de Análises Sociais e Economicas) realizou uma pesquisa para avaliar o perfil nutricional das familias beneficiarias do PBF em 229 municipios brasileiros em 2007. Nessa pesquisa foi observado que houve aumento no consumo de feijões (59\%), frutas (55\%) e vegetais $(40 \%) .{ }^{19}$ 
No (Gráfico2) estão os Marcadores de Alimentos Não Recomendáveis: Hambúrguer e/ou embutidos (33,53\%), macarrão instantâneo, salgadinho de pacote ou biscoito $(44,31 \%)$, biscoito recheado, doces ou guloseimas $(28,14 \%)$ e bebidas adoçadas $(61,68 \%)$ no ano de 2016 , já de acordo com o ano de 2017, apresentou 32,2\%, 33,17\%, 28,78\% e $56,1 \%$ nessa ordem respectivamente.

Gráfico2 - Distribuição percentual dos indivíduos adultos cadastrados pelo PBF e pelo SISVAN que referiam consumo conforme Marcadores de Alimentos não recomendáveis, no município de Dourados - MS, no ano de 2016 e 2017.

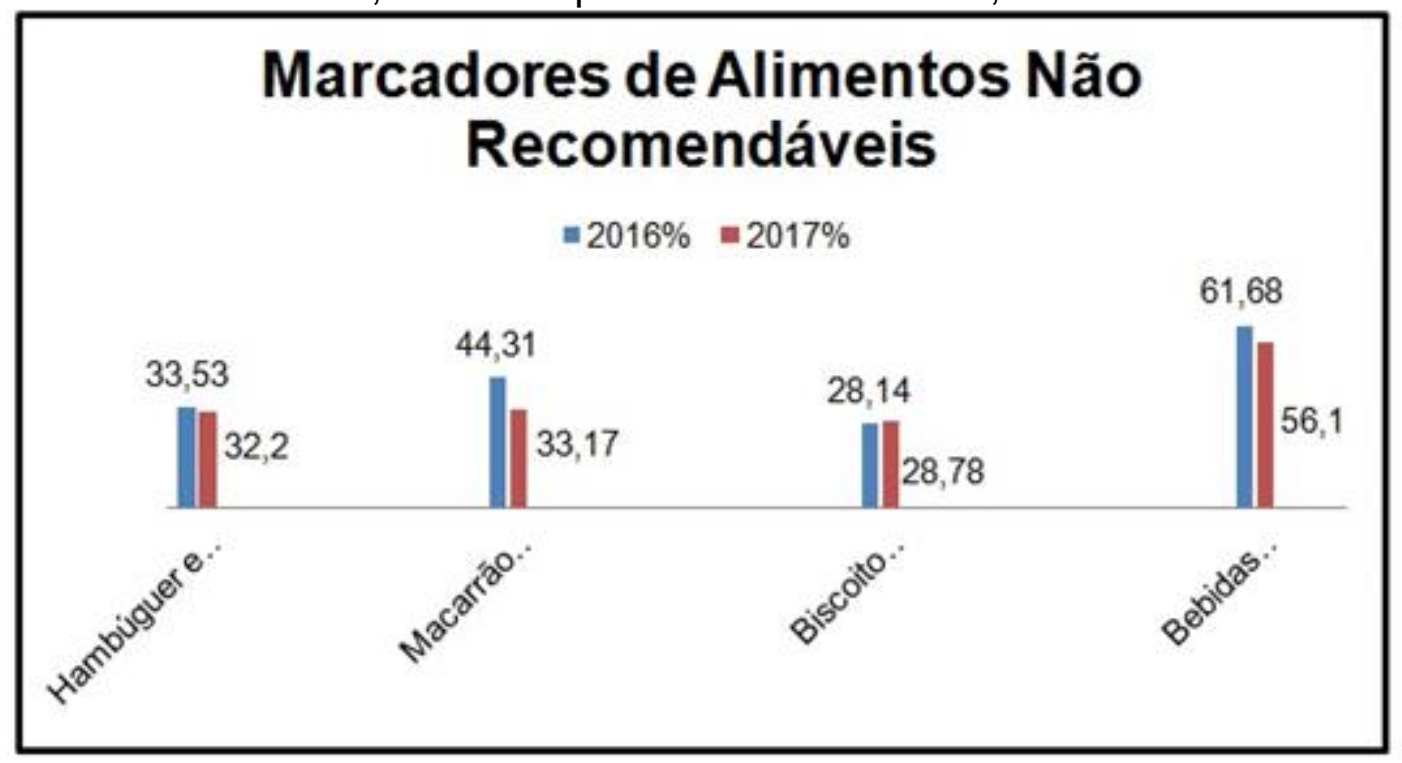

Fonte: Autores.

De acordo com os valores da figura 4, foi identificado à diminuição do consumo de hambúrguer e/ou embutidos em 1,33\%, macarrão instantâneo em $11,14 \%$.Já os salgadinhos de pacote ou biscoito, notaram-se um aumento do consumo em $0,64 \%$. Houve aumento no consumo de biscoito recheado, doces ou guloseimas no ano de 2017.

De acordo com dados do Vigitel (2016), no qual avaliaram 53.210 mil pessoas com mais de 18 anos nas capitais do país, observou-se que houve diminuição no consumo de refrigerantes ou suco artificial, sendo que no ano de 
2007 o consumo era de $30,9 \%$, passando para $16,5 \%$ no ano de 2016 , esse consumo era realizado pelo menos 5 dias ou mais na semana. ${ }^{15}$

O estudo de transição nutricional no Brasil desenvolvido em 2010 teve como objetivo realizar uma avaliação dos principais componentes da transição nutricional ocorrida no Brasil e suas consequências na população. Foi observado o aumento no consumo de ácidos graxos saturados, açúcares, refrigerantes, produtos industrializados com excesso de ácidos graxos "trans", guloseimas como doces, chocolates, balas, etc. Esses valores demonstram que ocorreram alterações no padrão alimentar da população, eles estão escolhendo alimentos mais calóricos, levando há um grande aumento no sobrepeso e obesidade da população em geral. ${ }^{16}$

Em 2014 uma pesquisa envolvendo 72 indivíduos,com o objetivo de diagnosticar a situação de insegurança alimentar e nutricional entre beneficiários do PBF de Montes Claros - MG, demonstrou o consumo inadequado do grupo dos doces e gorduras. $\mathrm{O}$ grupo dos doces apresentou $\mathrm{O}$ consumo de aproximadamente $51,7 \%$ e o grupo das gorduras apresentou 0 consumo de aproximadamente $51,7 \%{ }^{14}$

Ainda, segundo o IBASE, dos 5 mil entrevistados titulares do cartão Bolsa Família, em 229 municípios brasileiros do Nordeste, Centro-Oeste, Norte, Sudeste e Sul, no mês de setembro e outubro de 2007, foi observado que houve aumento na compra do grupo dos açúcares (78\%), biscoitos (63\%), alimentos industrializados (62\%) e óleos (55\%), mostrando alterações no padrão alimentar desta população. Esse padrão alimentar diário contribui para altas prevalências de sobrepeso e obesidade. ${ }^{19}$ 
Portanto, de acordo com os autores citados no texto pode-se observar um grande aumento no consumo de alimentos industrializados, doces, guloseimas, biscoitos de pacote, bebidas adoçadas. Esses alimentos consumidos diariamente e em altas quantidades podem levar ao aumento e a prevalência de sobrepeso e obesidade

\section{CONCLUSÃO}

A população avaliada no estudo apresentou altas taxas de sobrepeso e obesidade tanto no ano de 2016 como no ano de 2017, também demostrou o aumento do consumo de doces, guloseimas, bebidas adoçadas, hambúrguer e/ou embutidos e a diminuição do consumo de feijão, frutas e legumes e verduras quando comparados entre si.

A comprovação da transição nutricional provocou várias alterações ao longo do tempo e que estão presentes no dia a dia. Houve a diminuição de casos de desnutrição e o aumento de sobrepeso e obesidade, independente da idade, sexo, ou classe social da população. Devido essa transição nutricional e as mudanças que provocaram na população é preciso determinar estratégias de ação de saúde pública para combater esse grande aumento de casos de sobrepeso e obesidade no país.

Por isso, é de extrema importância que as áreas de atenção básica e saúde pública, trabalhem juntas e realizem estratégias para intervir nesse processo de transição que vem ocorrendo em todo país e especificamente nessa população avaliada. 


\section{REFERÊNCIAS BIBLIOGRÁFICAS}

1. PERES, Emanuela; FREITAS, Cibelly. Estado nutricional dos beneficiários do programa Bolsa Família no município de Sobral, Ceará, BRASIL. Sanare Revista de Políticas Públicas, Sobral, v.7, n.1, p.56-63, 2008. Disponível em: https://sanare.emnuvens.com.br/sanare/article/view/55, acesso em: 10/05/2108.

2. BRASIL. Ministério da Saúde. Secretaria de Atenção à Saúde. Departamento de Atenção Básica. Coordenação- Geral de Política de Alimentação e Nutrição. Manual de orientações sobre o bolsa família / Ministério da Saúde, Secretaria de Atenção à Saúde, Departamento de Atenção Básica, Coordenação - Geral de Política de Alimentação e Nutrição. - 1 ed. - Brasília: Ministério da Saúde, 2005. 32 p.

3. COTTA, Rosangela Minardi Mitre; MACHADO, Juliana Costa. Programa Bolsa Família e segurança alimentar e nutricional no Brasil: revisão crítica de literatura. Rev Panam Salud Publica. Minas Gerais, v.33, n.1, p.54-60, 2013. Disponível em: https://scielosp.org/pdf/rpsp/2013.v33n1/54-60/pt, acesso em: 10/05/2108.

4. SILVA, M. O. S. e. O Bolsa Família: problematizando questões centrais na política de transferência de renda no Brasil. Ciência \& Saúde Coletiva, Rio de Janeiro, v. 12, n. 6, p. 1429-1439. 2007. 
5. SECRETARIA MUNICIPAL DE ASSISTÊNCIA SOCIAL. Departamento de Proteção Social Básica. Bolsa família: contribuindo para combater a pobreza e desigualdade no Brasil. Departamento de Proteção Social Básica, Núcelo de Gestão de Benefícios Socioassistenciais. Prefeitura de Dourados-MS. p.3 -15. 2016.

6. ESCOTT-STUMP, Sylvia; MAHAN, L. Kathleen. Krause alimentos, nutrição \&dietoterapia. 13. ed. Rio de Janeiro: Elsevier; São Paulo: Roca, 2012, 1228p.

7. BRASIL. Ministério da Saúde. Saúde. Secretaria de Atenção à Saúde. Departamento de Atenção Básica. Coordenação-Geral de Alimentação e Nutrição. Marco de referência da vigilância alimentar e nutricional na atenção básica. Brasília, 2015a, 56p. Disponível em: http://bvsms.saude.gov.br/bvs/publicacoes/marco referencia vigilancia aliment ar.pdf, acesso em: 23/04/2018.

8. BRASIL. Ministério da Saúde. Secretaria de Atenção à Saúde. Departamento de Atenção Básica. Programa Bolsa Família. 2017. Disponível em: <http://dab.saude.gov.br/portaldab/ape_bfa. php, acesso em: 24/04/2018.

9. LEITE, Fabio.; BIN, Marcia.; SCHMITZ, Wanderlei. Produção do artigo científico. Faculdade de ciências e da saúde. Dourados MS: UNIGRAN, v.3, n.1. p.15-204, 2015. 
10. Organização Mundial da Saúde (1995 1997). Disponível em: https://arquivos.sbn.org.br/equacoes/eq5.htm., acesso em 09/10/2019

11. BARBETA, P.A. Estatística aplicada às ciências sociais. 5.ed. Florianópolis: UFSC. 2002. 339p.

12. LIMA, Flavia; RABITO, Estela; DIAS, Márcia. Estado nutricional de população adulta beneficiária do programa Bolsa Família no município de Curitiba-PR. Revista Brasileira Epidemiologia, Curitiba-PR, v.14, n.2. p.198206 , 2011. Disponível em: https://www.scielosp.org/scielo.php?pid=S1415790X2011000200002\&script=sci _arttext\&tlng=e , acesso em: 10/05/2018.

13. CABRAL, Marcela Jardim; VIEIRA, Karlla Almeida; SAWAYA, Ana Lydia; FLORÊNCIO, Telma Maria Menezes Toledo. Perfil socioeconômico, nutricional e de ingestão alimentar de beneficiários do Programa Bolsa Família. Estudos Avançados, São Paulo, v. 27, n. 78, p. 71-87. 2013. Disponível em:http://www.scielo.br/scielo.php?script=sci_arttext\&pid=S0103401420130002 00006, acesso em: 23/11/2018.

14. MONTEIRO, Elma Lúcia de Freitas; SOUZA, Cassília Rodrigues de; PINHO, Lucinéia de. Diagnóstico de Insegurança Alimentar e Nutricional entre beneficiários do Programa Bolsa Família participantes de oficina de intervenção nutricional. Segurança Alimentar e Nutricional, Campinas, v. 21, n. 2, p. 469- 
480 ,

2014.

Disponível

em:

https://periodicos.sbu.unicamp.br/ojs/index.php/san/article/view/8634476/2399, acesso em: 23/10/2018.

15. BRASIL. Ministério da Saúde. Secretaria de Atenção à Saúde. Departamento de Atenção Básica. Vigitel. 2016. Disponível em: http://portalarquivos.saude.gov.br/images/pdf/2017/abril/17/Vigitel.pdf, acesso em: 25/05/2018.

16. SOUZA, Elton Bicalho de. Transição nutricional no Brasil: análise dos principais fatores. Cadernos UniFOA. Volta Redonda, Ano V, n. 13 . p.49-53. 2010. Disponível

em: http://revistas.unifoa.edu.br/index.php/cadernos/article/view/1025/895, acesso em: 16/10/2018.

17. SILVANI, Juliana; BUSS, Caroline; PENA, Geórgia das Graças; RECCHI, Andrea Fontoura; WENDLAND, Eliana Márcia. Consumo alimentar de usuários do Sistema Único de Sáude segundo o tipo de assistência e participação no Bolsa Família. Ciência \& Saúde Coletiva. Rio de Janeiro, v.23, n.8, p.2599 2608. 2018. Disponível em: http://www.scielo.br/scielo.php?script=sci_arttext\&pid=\$1413812320180008025 99\&lng=pt\&tlng=pt\#B6, acesso em: 16/10/2018.

18. SPERANDIO, Naiara; RODRIGUES, Cristiana Tristão; FRANCESCHINI, Sylvia do Carmo Castro; PRIORE, Silvia Eloiza. Impacto do Programa Bolsa 
Família no consumo de alimentos: estudo comparativo das regiões Sudeste e Nordeste do Brasil. Ciência \& Saúde Coletiva. Rio de Janeiro, v, 22, n. 6, p.1771-1780. 2017. Disponível

em: http://www.scielo.br/scielo.php?script=sci_arttext\&pid=\$1413812320170026017 71\&lng=pt\&tlng=pt\#B20, acesso em: 16/10/2018.

19. INSTITUTO BRASILEIRO DE ANÁLISES SOCIAIS E ECONÔMICASIBASE. Repercussões do Programa Bolsa Família na segurança alimentar e nutricional das famílias beneficiadas. Documento Síntese PBF. Disponível em: http://www.ibase.br/userimages/ibase_bf_sintese_site.pdf, acesso em: 23/11/2018.

20. MINISTÉRIO DA SAÚDE. Informação e Gestão da Atenção Básica. 2017. Disponível em: https://egestorab.saude.gov.br/, acesso em: 9/10/19. 


\section{APÊNDICE A - Termo de Guarda \\ TERMO DE COMPROMISSO PARA USO DE DADOS/MATERIAL}

As pesquisadoras do presente projeto, Dayane Ribeiro Dos Santos e Patrícia Cintra, intitulado: "AVALIAÇÃO DO ESTADO NUTRICIONAL DOS BENEFICIÁRIOS ADULTOS DO PROGRAMA BOLSA FAMÍLIA NACIDADE DE DOURADOS-MS" se comprometem a preservar a privacidade dos pacientes cujos dados antropométricos e de consumo alimentar serão coletados em relatórios no sistema do Sisvan de indivíduos cadastrados no Bolsa Família e no Sisvan web do ano de 2016 e 2017.

Concordam, igualmente, que estas informações serão utilizadas única e exclusivamente para execução da presente pesquisa. As informações somente poderão ser divulgadas de forma anônima.

Dourados, 11 de Maio de 2018.

Pesquisador Responsável

Comitê de Ética em Pesquisa com Seres Humanos Centro Universitário da Grande Dourados - UNIGRAN

R: Balbina de Matos, 2121 - Fone: (67) 3411-4207 / Fax: 3411-4167 CEP: 79824-900 - Dourados - MS.E-mail: comitedeetica@unigran.br 